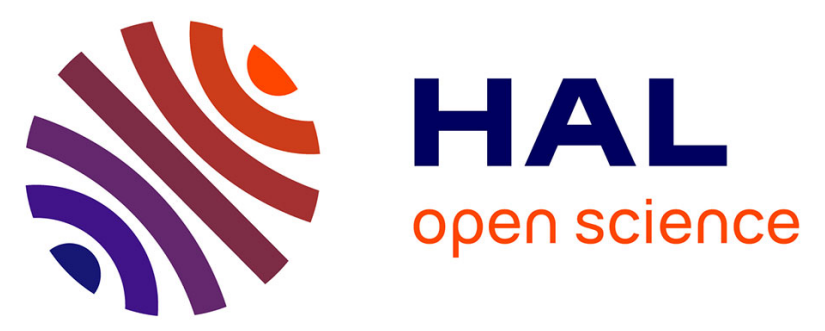

\title{
PASITHEA: An Integrated Monitoring and Therapeutic System for Sleep Apnea Syndromes Based on Adaptive Kinesthetic Stimulation
}

A I. Hernández, G Guerrero, D Feuerstein, L Graindorge, D Perez, A Amblard, P Mabo, J-L Pépin, Lotfi Senhadji

\section{To cite this version:}

A I. Hernández, G Guerrero, D Feuerstein, L Graindorge, D Perez, et al.. PASITHEA: An Integrated Monitoring and Therapeutic System for Sleep Apnea Syndromes Based on Adaptive Kinesthetic Stimulation. Innovation and Research in BioMedical engineering, 2016, 37 (2), pp.81-89. 10.1016/j.irbm.2016.02.009 . hal-01289821

\section{HAL Id: hal-01289821}

https://hal-univ-rennes1.archives-ouvertes.fr/hal-01289821

Submitted on 17 Mar 2016

HAL is a multi-disciplinary open access archive for the deposit and dissemination of scientific research documents, whether they are published or not. The documents may come from teaching and research institutions in France or abroad, or from public or private research centers.
L'archive ouverte pluridisciplinaire HAL, est destinée au dépôt et à la diffusion de documents scientifiques de niveau recherche, publiés ou non, émanant des établissements d'enseignement et de recherche français ou étrangers, des laboratoires publics ou privés. 


\title{
PASITHEA : an integrated monitoring and therapeutic system for sleep apnea syndromes based on adaptive kinesthetic stimulation
}

\author{
A. I. Hernández ${ }^{a, b}$, G. Guerrero ${ }^{a, b}$, D. Feuerstein ${ }^{c}$, L. Graindorge ${ }^{c}$, D. \\ Perez $^{\mathrm{a}, \mathrm{b}}$, A. Amblard ${ }^{\mathrm{c}}$, P. Mabo ${ }^{\mathrm{a}, \mathrm{b}, \mathrm{d}}$, J-L. Pépin ${ }^{\mathrm{e}}$, L. Senhadji ${ }^{\mathrm{a}, \mathrm{b}}$ \\ ${ }^{a}$ INSERM, U1099, Rennes, F-35000, France. \\ ${ }^{b}$ Université de Rennes 1, LTSI, Rennes, F-35000, France. \\ ${ }^{c}$ Sorin CRM SAS (a LivaNova company), Clamart, France. \\ ${ }^{d}$ CHU Rennes, Department of Cardiology Rennes, F-35000, France. \\ ${ }^{e}$ Univ Grenoble Alpes, CHU Grenoble, Sect Physiol Sommeil \&3 Exercice, Inserm U1042, \\ Pole Thorax \& Vaisseaux, Lab EFCR, F-38000 Grenoble, France
}

\begin{abstract}
Sleep apnea syndromes are characterized by repeated episodes of breathing pauses (apnea) or significant reductions in respiratory amplitude (hypopnea) during the patient's sleep. These episodes significantly alter the sleep structure of the patient and provoke acute cardiorespiratory responses that may be deleterious in the long term. Continuous positive airway pressure (CPAP) therapy is the gold-standard treatment for SAS, but patient compliance to this therapy is low. This paper presents a new system for real-time detection, monitoring and treatment of SAS, based on triggered kinesthetic stimulation. The system is composed of 3 components: i) a cardio-respiratory recorder, ii) a kinesthetic stimulator and iii) a real-time processing application, integrating an "on-off" control method to trigger the kinesthetic stimulation as a function respiratory event detections. A preliminary evaluation of the system, performed on 46 patients suffering from severe SAS is also presented. This evaluation is focused on i) the performance characterization of the realtime respiratory event detector and ii) a qualitative description of the effect of the therapy. A satisfactory real-time respiratory event detection performance $($ sensitivity $=89.2 \%$, positive predictive value $=59 \%$ ) was obtained with the proposed detector, when evaluated retrospectively using the acquired data. Three case reports, describing the cardiorespiratory response to the therapy of a responder, a partially-responder and a non-responder patients are
\end{abstract}


also presented. These preliminary results are very encouraging and warrant further developments, in particular for novel control stimulation strategies.

Keywords: Sleep apnea syndrome, kinesthetic stimulation, closed-loop therapy

\section{Introduction}

Sleep apnea syndrome (SAS) is a multifactorial disease characterized by repeated episodes of breathing pauses (apnea) or significant reductions in respiratory amplitude (hypopnea) during the patient's sleep. SAS concerns more than $5 \%$ of the general population, but it is still under-diagnosed $[1,2]$. This is particularly true in populations considered "at risk", such as those suffering from cardiovascular diseases and type 2 diabetes, where the presence of SAS increases the risk of complications $[3,4,5]$. In these populations, in particular, the prevalence of SAS is estimated to be greater than $50 \%$ [6].

Patients suffering from SAS show apnea or hypopnea episodes that can be repeated from 5 to 100 times per hour of sleep, with durations ranging from 10 seconds to several minutes, strongly affecting the sleep structure of the patient. The "apnea hypopnea index" (AHI), calculated as the number of apnea and hypopnea events per hour, is a key clinical marker to quantify the severity of apnea in a given patient. The gold-standard for SAS diagnosis is polysomnography (PSG), which requires manual scoring of all events by a trained specialist. Consequently, SAS remains highly underdianosed due to long waiting-lists in sleep laboratories. There is therefore the need for new reliable automatic diagnostic strategies for improving the detection and management of patients suffering from SAS [7]. Diagnostic systems should include more advanced data processing methods, able to better exploit the complementary information acquired from different cardiorespiratory signals. Regarding therapy, the gold-standard treatment is by continuous positive airway pressure (CPAP). The CPAP machine delivers a continuous stream of compressed air via a nose or full-face mask. This positive pressure keeps the airway open and prevents it from collapsing, so that unobstructed breathing becomes possible [8]. However, patient compliance is low, with $15 \%$ initial refusal and low adherence in the long term for 20-30\% of them [9]. Therefore, new therapeutic solutions are also needed.

This paper describes the first version of a new system for the detection, monitoring and treatment of SAS, directed to improve patient management 
by integrating both a real-time diagnostic approach and a better treatment tolerance that may improve long-term observance. This system has been developed within the framework of the ANR TecSan project entitled "Personalized and Adaptive kinesthetic StImulation Therapy, based on cardiorespiratory Holter monitoring, for slEep Apnea syndromes" (PASITHEA), supported by the French "Agence Nationale pour la Recherche". Preliminary results from the first clinical evaluation (HYPNOS study) of the system will be presented and discussed.

\section{System description}

The PASITHEA system is composed of three main components (Fig. 1):

1. a cardiorespiratory ambulatory recorder (Holter), providing data acquisition and wireless transmission of the acquired data in real time;

2. a kinesthetic stimulation system, comprising a stimulation signal generator and a kinesthetic actuator, typically positioned behind the ear;

3. a control device for adaptive kinesthetic stimulation which will be embedded, in a first phase, into a computer.

These three components interact using a specific wireless communication protocol, based on the Bluetooth (BT) technology. Eight complete systems were built and tested. The following subsections describe each of the main modules of the system in more details.

\subsection{Cardiorespiratory Holter}

A commercially available Sorin Holter system was modified by partner SORIN CRM in order to meet the requirements of the project. The new cardiorespiratory Holter prototype is capable of acquiring and locally recording two ECG channels (sampling frequency: $256 \mathrm{~Hz}$, resolution: $10 \mu \mathrm{V}$, frequency response: 0.05-80 Hz), nasal pressure (sampling frequency: $256 \mathrm{~Hz}$ ) and blood oxygen saturation $\left(\mathrm{SaO}_{2}\right)$ (sampling frequency: $1 \mathrm{~Hz}$ ) during a whole night. This system can also transfer these data in real-time (every 100 ms) through a BlueTooth Low Energy (BTLE) link towards the real-time signal processing application for further processing.

\subsection{Kinesthetic stimulation system}

The kinesthetic stimulation system has been developed by partner LTSI, based on previous prototypes already used for kinesthetic stimulation therapy 


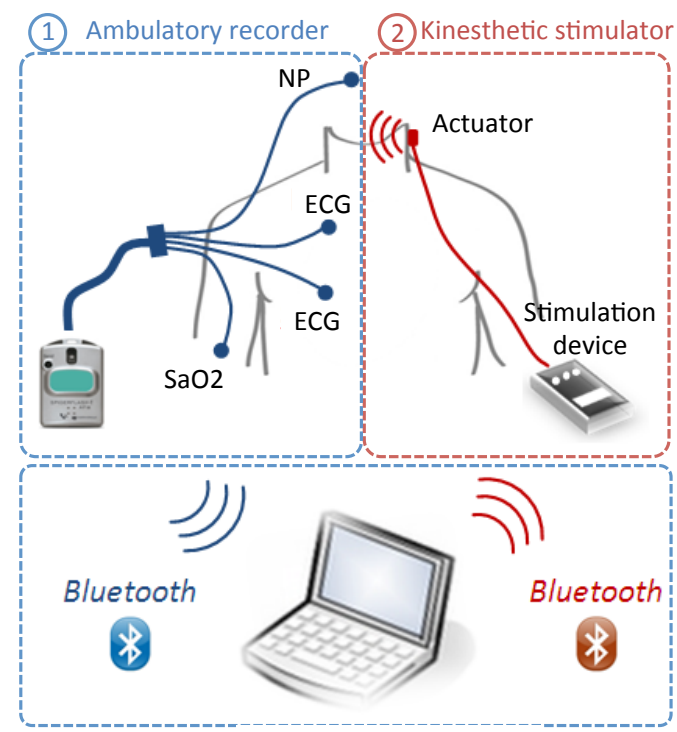

(3) Real-time control

Figure 1: General diagram of the PASITHEA system showing its main components: 1) the cardio-respiratory Holter, 2) kinesthetic stimulator and 3) the real-time application for data processing and control.

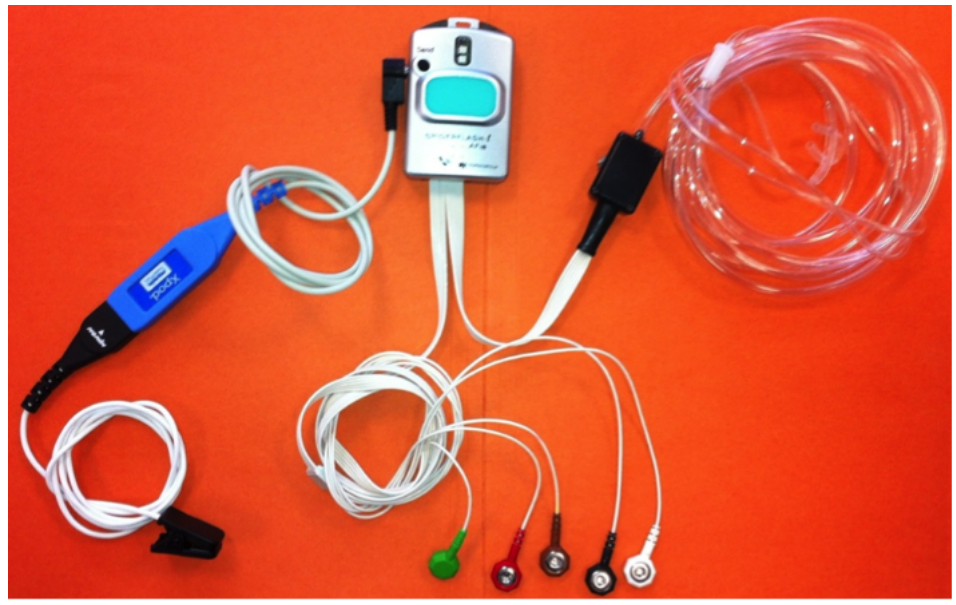

Figure 2: The prototype cardiorespiratory Holter device with associated sensors (ECG electrodes, $\mathrm{SaO}_{2}$ ear sensor and nasal pressure cannula). 
for apnea-bradycardia on preterm newborns [10]. The system consists in a command module (Figure 3a) and a kinesthetic actuator (Figure 3b).

The command module is a battery-operated device that can generate dynamic stimulation signals in an adaptive fashion. This module is based on an ATmega168 micro-controller, embedding a custom, multi-agent software that handles all the functionalities of the device. In particular, the micro-controller can synthesize sinus signals with varying amplitudes and frequencies, to be presented as input to a driver circuit that will handle the kinesthetic effector. The command module can function either in an "autonomous" or a "slave" mode. The slave mode requires establishing a BT connection with a master system (in this case, the real-time control application) that will command the operation of the stimulator.

The stimulating device is a resonant linear actuator with optimal resonance frequency of $175 \mathrm{~Hz}$ (model C10-100, Precision Microdrives Ltd, London, UK). At 100\% of the acceptable input signal amplitude (2V RMS), this actuator generates a typical normalized acceleration of $13.7 \mathrm{~m} / \mathrm{s}^{2}$.

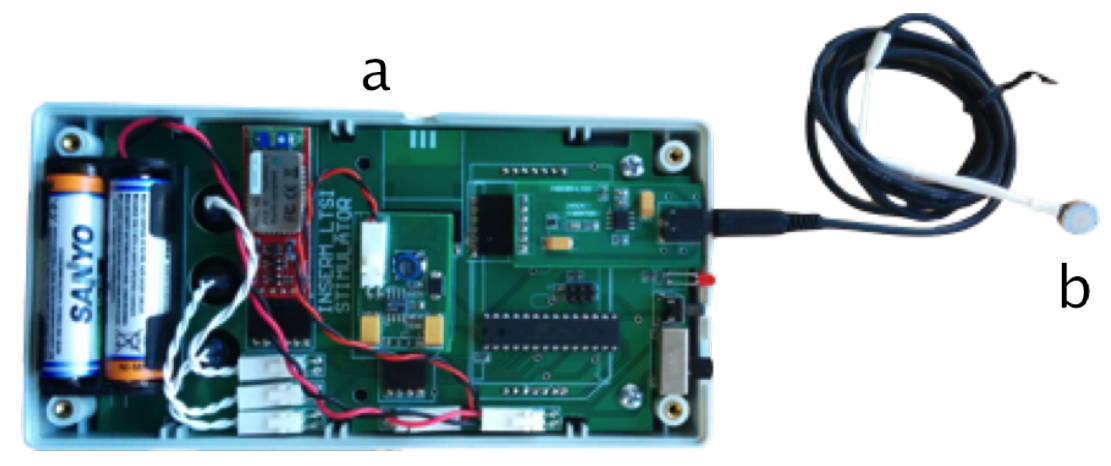

Figure 3: The kinesthetic stimulation system, showing (a) an opened control module and (b) and the kinesthetic actuator.

\subsection{Real-time processing and control application}

This application, depicted in figure 4, is in charge of: i) establishing wireless communication links with the Holter and the stimulator, ii) receiving data from the cardio-respiratory Holter, iii) performing real-time signal processing on the received signals for the detection of apnea or hypopnea episodes and iv) according to the detector's output, apply the proposed "on- 
off" controller and send the appropriate command to the kinesthetic stimulator through a BT link.

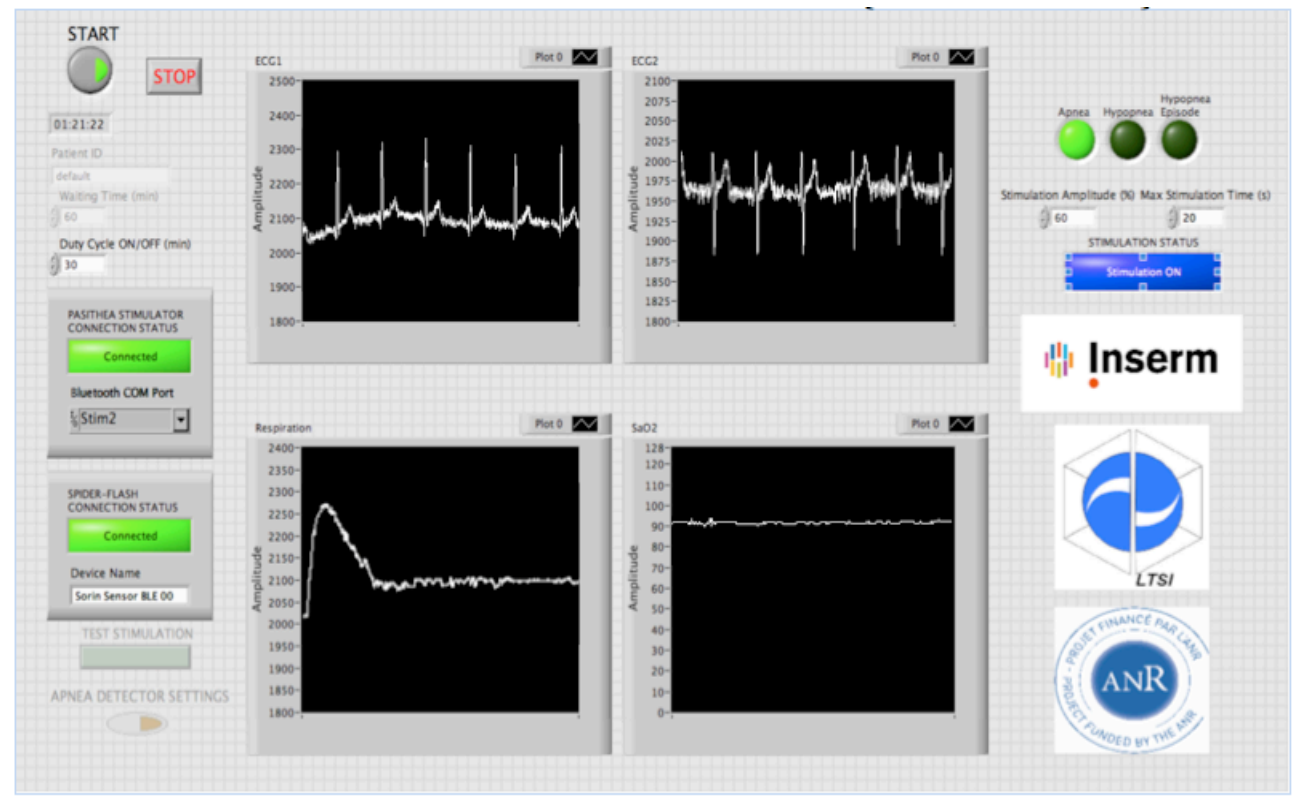

Figure 4: Real-time application for data acquisition, processing and control. Left panel: user-defined configuration parameters and BT connexion with the Holter and the stimulator. Central panel: 4 screens showing real-time acquired data: 2 ECG channels (top), nasal pressure (bottom left), $\mathrm{SaO}_{2}$ (bottom right). Right panel: Output of the real-time respiratory event detector (apnea or hypopnea) and stimulation parameters.

\subsubsection{Wireless communications and data acquisition:}

The control application establishes wireless BT links with the Holter and the stimulator device and performs device initialization and management using specifically developed application-level communication protocols. Methods of error correction and verification of the integrity of the BT links have been integrated into the protocol and are also handled by the control application. Data acquisition is initiated and terminated by the application and all available signals are received in real-time from the Holter at their original sampling rates. 


\subsubsection{Real-time respiratory event detector}

The respiratory event detector is based on the segmentation of respiratory cycles from the acquired nasal pressure signal. A full respiratory cycle is defined as inhalation followed by exhalation. Inhalation is detected when the nasal pressure signal exceeds a positive threshold value $\left(\lambda_{I}(t)\right)$. Exhalation is detected when nasal pressure signal falls below a negative threshold value $\left(\lambda_{E}(t)\right)$. Thresholds $\lambda_{I}(t)$ and $\lambda_{E}(t)$ are updated independently, through time, according to the patient's breathing patterns. An apnea is detected as the absence of inhalation for more than $\delta_{a} \in[3, \ldots, 10]$ seconds. A hypopnea event is detected when inhalation amplitude falls below $50 \%$ below inhalation baseline for more than $\delta_{h} \in[5, \ldots, 10]$ seconds. A complete description, as well as a prospective evaluation of this method can be found on a recent publication [11]. In this paper, we propose an improvement of this detector, integrating the following modifications:

- an additional criterion was required for apnea detection: an apnea was detected not only as the absence of inhalation for a certain time but also as a "flat" breathing line, i.e. nasal pressure amplitude had to stay within the inhalation and exhalation thresholds

- the time to detect apneas $\left(\delta_{a}\right)$ was not a fixed parameter but would be set, within limits, as the average of the patient's latest respiratory cycle periods.

- the time to detect hypopnea $\left(\delta_{h}\right)$ was not a fixed parameter but linked to the amplitude of inhalation drop: a quicker detection for severe hypopnea (with a large drop) and a slower detection for less severe hypopnea

A retrospective assessment of this improved version (referred to as v2) is given in Table 1. However, it is to note that the output of the original version of the detector has been used as control variable for triggering kinesthetic stimulation for the patients included in the Hypnos study.

\subsubsection{Control of the kinesthetic stimulation}

In this first version of the PASITHEA system a real-time "on-off" control method is implemented into the application, using as control variable the detector's output. When respiratory event detection is confirmed, around 8 seconds after the beginning of the respiratory event, a command is sent 
to the kinesthetic stimulator to activate it. Although the system is able to apply different stimulation strategies for different types of respiratory events $[12,13]$, the following unique stimulation strategy was used in this paper: A constant amplitude is applied to the patient at a frequency of $175 \mathrm{~Hz}$. Kinesthetic stimulation is organized into bursts of a maximum duration of $3 \mathrm{~s}$ each, followed by a silent period of $2 \mathrm{~s}$. A maximum of 3 bursts are applied in a given detected respiratory event. This burst-based stimulation is applied in order to minimize patient habituation to the stimulation as well as the fragmentation of the patient's sleep. When the detector confirms the end of the respiratory event (first detected deflection on the nasal pressure signal above a given threshold during a respiratory event) a command is sent to stop kinesthetic stimulation.

\section{System evaluation methodology}

Evaluation of this first version of the PASITHEA system has started within the framework of a clinical protocol approved by the ethics committee of the Grenoble University Hospital (HYPNOS study), and involving five centers: the University Hospitals of Angers, Grenoble, Montpellier, Rennes and Tours. The first objective of this study is to validate the on-line real-time methods for the detection and characterization of apnea and hypopnea during sleep, taking the polysomnography (PSG) as a gold standard. A secondary objective of this study is to obtain preliminary results on the effect of the proposed on-off controlled kinesthetic stimulation on respiratory events.

\subsection{Data acquisition and patient population}

After signing an informed consent, all patients underwent a full PSG in a sleep laboratory. Nasal pressure (NP), electrocardiography (ECG) and oxygen saturation $\left(\mathrm{SaO}_{2}\right)$ were simultaneously acquired and processed by the PASITHEA system. Using a T-deviation tube, the same nasal pressure was presented as input to the sensors of both the cardiorespiratory Holter and the PSG systems. Central reading of PSG was performed by an expert, blinded core-lab (CHU Grenoble, France) who provided the overall AHI together with an accurate scoring of individual events (start, duration and type of event) for each patient.

A total of 46 severe obstructive sleep apnea (OSA) patients (mean age: 58.5, BMI: $30.1 \mathrm{~kg} / \mathrm{m} 2$, AHI: $47.7 / \mathrm{h}$ - with $92 \%$ of obstructive events, $75 \%$ male) were included in this study. 12 patients were included in a titration 
phase, dedicated to optimize the parameters of the respiratory event detector and to establish the amplitude of the kinesthetic bursts that were used for the rest of the study. 34 additional patients were included on the study phase, with a fixed stimulation amplitude of $80 \%$ of the maximum power of the stimulator (normalized acceleration of approx. $11 \mathrm{~m} / \mathrm{s}^{2}$ ). This stimulation amplitude value was determined during the titration phase. 4 patients were totally excluded from the analysis due to problems with the PSG recording, or to a bad nasal cannula signal, preventing correct detection of respiratory events. Additionally, 4 other patients are excluded from the therapy analysis because the stimulation therapy was not delivered (failure of the stimulator cable or connector), but they can participate in the analysis of the detection performance. In total, 30 patients are thus available for the analysis of detection performance and 26 patients are available for the analysis of the therapy.

\subsection{Evaluation of the respiratory event detector}

Regarding the evaluation of the detector's performance, a training set constituted of the first 12 patients included in the study was used for the definition of the detector parameters. At the end of this phase, the parameters were fixed to $\delta_{a}=8$ seconds for the apneas and $\delta_{h}=10$ seconds for hypopnea (see section above). A test set composed of the remaining 30 patients was used to assess the detector performance. Performance was assessed by matching PSG annotated events (blinded core-lab scoring) with the PASITHEA detector output. A true positive (TP) event was defined as an event detected less than 10 seconds before or within the duration of a PSG-annotated event. Note that for a global assessment of respiratory event detection, apneas detected as hypopneas (and reciprocally) were regarded as true positives. Delay to the detection was defined as the time lag between the detector output and the start of the event, as per core-lab PSG annotation. The detector sensitivity (SE) and positive predictive value (PPV) were calculated as usual:

$$
\begin{gathered}
S E(\%)=T P /(T P+F N) \cdot 100 \\
P P V(\%)=T P /(T P+F P) \cdot 100
\end{gathered}
$$

where FN and FP represent respectively the number of false negative and false positive detections. The $95 \%$ confidence intervals of the detector outputs were calculated using the Wilson score. 


\subsection{Preliminary evaluation of the cardiorespiratory response to the therapy}

For this preliminary evaluation, each patient represented its own reference, by defining successive periods during which the simulator was active (stim-on) or inactive (stim-off). The first hour of the recording was performed without any stimulation in order to let the patient fall asleep. The rest of the recording was divided into consecutive segments of 30 minutes in stim-on and 30 min of stim-off.

A qualitative patient-by-patient analysis of the obtained cardiorespiratory responses to the kinesthetic therapy was performed, with the objective of evaluating if a given patient correctly responds to the kinesthetic stimulation. A correct response has been defined as a return to normal breathing, during or immediately after the delivery of kinesthetic stimulation. The absence of a return to normal breathing after the end of the third stimulation burst was considered as a no-response. A partial response was defined when the patient intermittently showed a correct or a no-response to the therapy throughout the night. Preliminary results shown in this paper will consist on three casereports regarding the cardiorespiratory response observed from i) a responder patient, ii) a partially-responder patient and iii) a non-responder patient.

\section{Results}

\subsection{Evaluation of the apnea/hypopnea detector}

The global performance of the real-time apnea/hypopnea detector is given in Table 1. Only the stim-off periods were included in the analysis, to avoid any bias of potential events interrupted by kinesthetic stimulation. Overall, 2561 apneas and 1679 hypopneas were scored on the PSG. 3656 events were correctly detected within the 10-second window (see definition of a true positive above). 2367 false positive events were detected, among which 318 apneas and 697 hypopnea were wrongly detected while the patient was awake. Breathing baseline fluctuations due to movements during awake periods explain the high number of wrongly detected hypopneas, while nasal cannula withdrawal and physiological apneas at sleep onset (that are not scored on the PSG since they are not pathological) account for most of the false positive apneas during wakefulness. The use of a sleep detector, that would temporarily turn off the real-time event detection while the patient is awake, would significantly improve the PPV (second line of Table 1).

Apnea and hypopnea events were always detected well before the conventional 10 seconds requirement to define a respiratory event, with a mean 
delay of 6.0 seconds and 8.7 seconds for apneas and hypopneas respectively. The second version of the detector (v2), when tested retrospectively on the test set significantly reduced this detection delay (down to 4.1 seconds for apneas and 3.5 seconds for hypopneas on average) while sensitivity slightly improved and PPV remained similar.

Table 1: Real-time respiratory event detector performances. Values are given as mean [95\% Confidence intervals]. The first two lines give the performances of the on-line detector, as assessed prospectively on all 30 patients included in the evaluation phase of the study. The last line gives the performances of the improved detector (v2) on the same patients, but assessed retrospectively.

\begin{tabular}{|c|c|c|c|c|}
\hline $\begin{array}{l}\text { Analysis } \\
\text { method }\end{array}$ & $\mathrm{SE}(\%)$ & $\operatorname{PPV}(\%)$ & $\begin{array}{c}\text { Apnea } \\
\text { detection } \\
\text { delay (sec) }\end{array}$ & $\begin{array}{l}\text { Hypopnea } \\
\text { detection } \\
\text { delay (sec) }\end{array}$ \\
\hline $\begin{array}{l}\text { Prospective } \\
\text { Event-based }\end{array}$ & $\begin{array}{c}86.2 \\
{[85.2-87.2]}\end{array}$ & $\begin{array}{c}60.7 \\
{[59.5-61.9]}\end{array}$ & 6.0 & 8.7 \\
\hline $\begin{array}{l}\text { Prospective } \\
\text { Event-based } \\
\text { Asleep only }\end{array}$ & $\begin{array}{c}86.2 \\
{[85.2-87.2]}\end{array}$ & $\begin{array}{c}78.3 \\
{[77.1-79.4]}\end{array}$ & 6.0 & 8.7 \\
\hline $\begin{array}{c}\text { Retrospective (v2) } \\
\text { Event-based }\end{array}$ & $\begin{array}{c}89.2 \\
{[88.4-89.8]}\end{array}$ & $\begin{array}{c}59.0 \\
{[58.1-59.9]}\end{array}$ & 4.1 & 3.5 \\
\hline
\end{tabular}

\subsection{Qualitative preliminary responses of the kinesthetic stimulator}

This section will present three case reports, describing the cardiorespiratory response to the proposed triggered kinesthetic therapy of a responder patient, a partially responder patient and a non-responder patient.

\subsubsection{Responder patient}

Figure 5 shows the cardio-respiratory effect of the therapy on one responder patient, using the PASITHEA system v1. The first $10 \mathrm{~min}$ in this figure correspond to a stim-off segment and the following 10 min correspond to a stim-on phase. The nasal pressure (NP) signal shown in the upper panel (Fig. 5-A) was applied as input to the above-mentioned respiratory event detector. 
Figure 5-B shows the stimulation signal (Stim), obtained as output from the on-off controller, used to activate the kinesthetic actuator. Note that, as expected, no stimulation is performed during the stim-off phase and different stimulation bursts are delivered during the stim-on phase. As described above, all delivered stimulations have the same amplitude and the number of bursts delivered depends on the duration of the respiratory event. For this patient, in most cases, the respiratory episode is stopped during the first stimulation burst. However, some over-stimulations were observed on this patient, due to a lack of a correct detection of the end of the event for some hypopnea episodes.

The first effect that can be noticed from the therapy in this responder patient is that the duration of the apnea events is smaller during the stim-on phase than during the stim-off phase. Also, due to these extended respiratory events, oxygen saturation $\left(\mathrm{SaO}_{2}\right)$ (Figure 5-C) shows significant hypoxia events (low oxygen saturation, some of them below 90\%) during the stimoff phase. These hypoxemia events are stopped by the stimulation therapy, during the stim-on phase. Figure 5-D shows the instantaneous heart rate (HR) during the same period. It can be noticed that tachycardia (high HR) events, correlated to the apnea and hypoxia events and provoked by chemo and baroreflex autonomic functions, are observed during the stim-off phase. These tachycardia episodes are not present during the stim-on phase, during which a normal and more stable respiratory sinus arrhythmia is observed. Also, the mean HR during the whole stim-off episode was higher than the mean HR of the stim-on episode. Finally, Figure 5-E shows the hypnogram reconstructed from the manual annotations performed by the Core-lab. The patient spent most of the time in this segment in REM and light sleep (LS) stages. A number of micro-arousals (MA) are observed during the segment, particularly at the end of each apnea episode during the stim-off phase and after most kinesthetic stimulations, during the stim-on phase. One "awake" event was observed during the stim-on phase. A first qualitative analysis of the sleep structure for this patient showed no major differences between the stim-on and stim-off phases.

\subsubsection{Partially responder patient}

As previously defined, partially responder patients show a correct response to the therapy during some parts of the night and a lesser or no response in other parts of the night. This loss of response was often associated with changes in posture or sleep stages during the night. Figure 6 depicts 

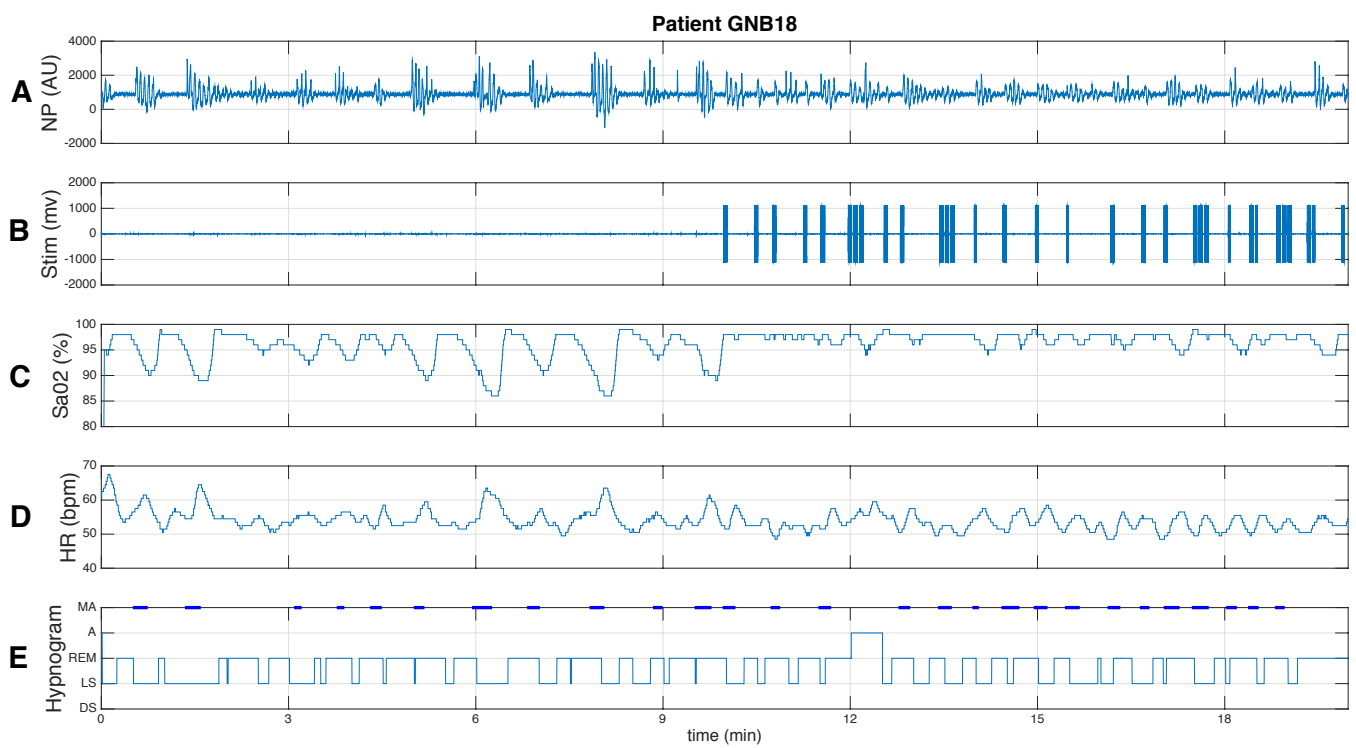

Figure 5: Cardiorespiratory response to kinesthetic stimulation of a responder patient. The first 10 minutes correspond to the end of a non-stimulation (stim-off) phase and the next 10 minutes to a phase with triggered stimulation active (stim-on). The following signals are displayed: A) nasal pressure (NP), B) signal driving the kinesthetic stimulator (Stim), C) oxygen saturation $\left(\mathrm{SaO}_{2}\right)$, D) instantaneous heart rate (HR), as detected from the ECG, E) Hypnogram obtained from Core-lab annotations ( $\mathrm{MA}=$ micro-arousal, A=Awake, $\mathrm{REM}=$ Rapid eye movement, $\mathrm{LS}=$ light sleep, $\mathrm{DS}=$ deep sleep). This response shows extended respiratory episodes accompanied by repeated hypoxia events during the stim-off phase. Respiratory event durations are reduced and no hypoxia events $\left(\mathrm{SaO}_{2}<90 \%\right)$ are observed during the stim-on phase. The stim-off phase is also characterized by significant tachycardia events that are related to sympathetic activations due to prolonged apnea and hypoxia. These tachycardia episodes are not observed during the stim-off phase, which shows a physiological respiratory sinus arrhythmia. 
the same signals shown in Figure 5, but for a partially responder patient. A correct response to the therapy is observed during the first minutes. The patient starts moving from a lateral to a dorsal position around minute 5. Once in stable dorsal position (around minute 10), the response to the therapy is strongly reduced, presenting, in particular, three respiratory episodes with the maximum stimulation of 3 burst that failed to restore normal breathing. As a consequence of this non-response, hypoxia episodes and tachycardia, which were not observed during the first 10 minutes, appear after minute 10 . This example suggests that i) the actuator should be carefully fixed to the skin of the patient and ii) stimulation parameters should be adjusted over time in order to improve response.
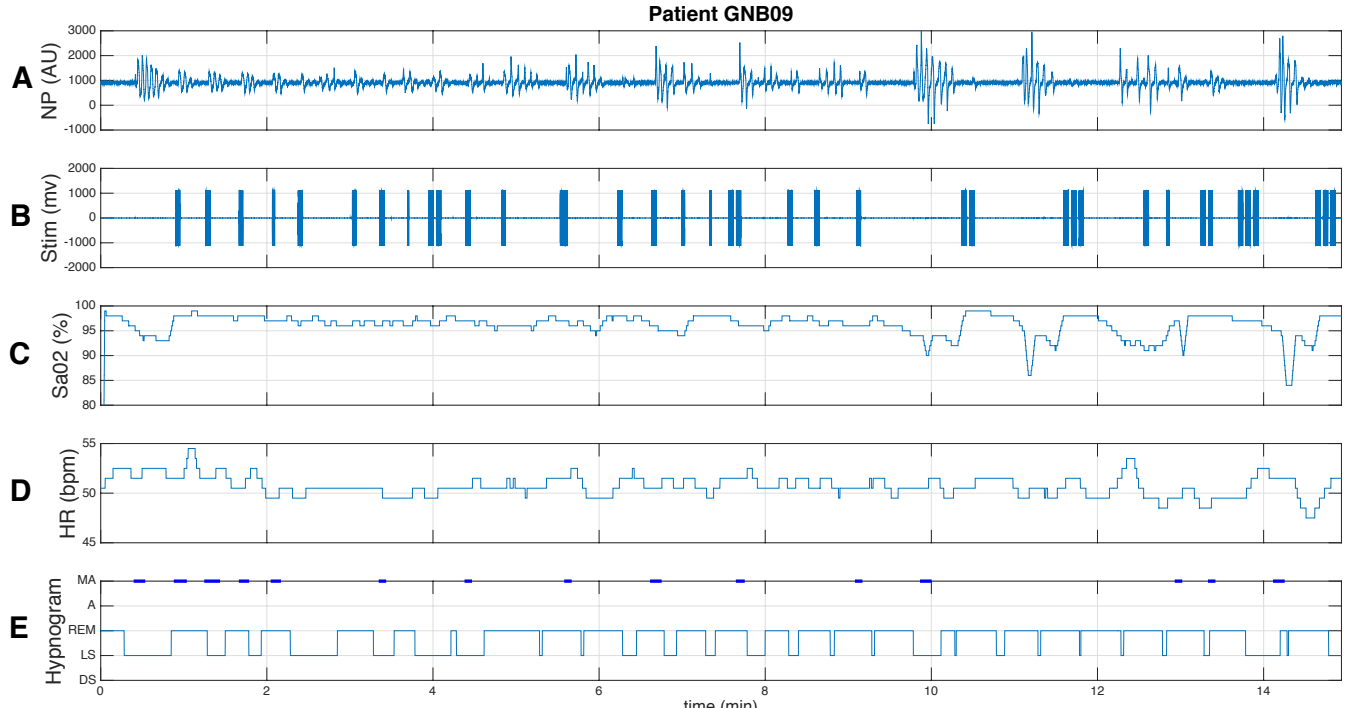

Figure 6: Cardiorespiratory response to kinesthetic stimulation of a partially responder patient. The whole segment was acquired during a stim-on phase. The following signals are displayed: A) nasal pressure (NP), B) signal driving the kinesthetic stimulator (Stim), C) oxygen saturation $\left(\mathrm{SaO}_{2}\right)$, D) instantaneous heart rate (HR), as detected from the ECG, E) Hypnogram obtained from Core-lab annotations ( $\mathrm{MA}=$ micro-arousal, $\mathrm{A}=$ Awake, $\mathrm{REM}=$ Rapid eye movement, $\mathrm{LS}=$ light sleep, DS = deep sleep). In this example, the patient responds correctly to the therapy during the first 9 minutes. At minute 6 the patient starts moving and changes to dorsal position in minute 10. In this new position (from minute 10), the effect of the therapy is reduced letting appear hypoxia and tachybradycardia episodes. 


\subsubsection{Non-responder patient}

Figure 7 presents an example of a non-responder patient. Most of the respiratory events in this example were treated by the maximum of 3 stimulation bursts and normal respiration was not recovered during or just after the end of the stimulation on each event. Even if the direct contribution of the stimulation therapy in this case is not clear, apnea durations on this segment are rather low, limiting the arrival of hypoxic events. Nevertheless, marked increases in HR and micro-arousals are observed after each major respiratory episode.
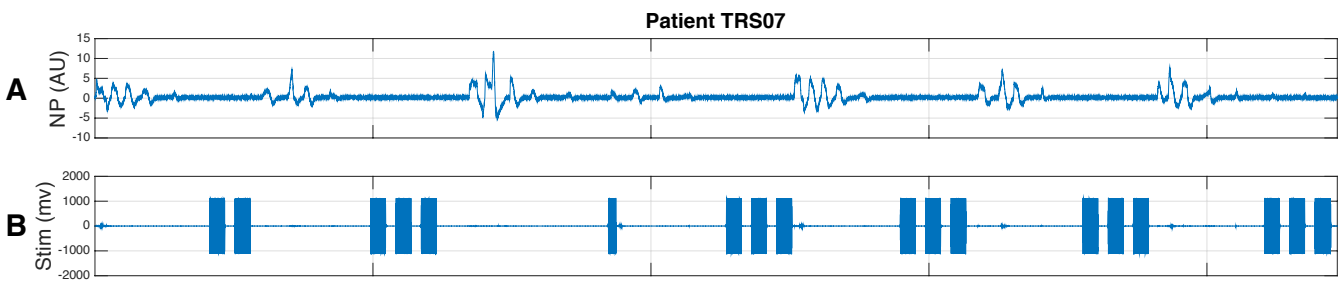

C
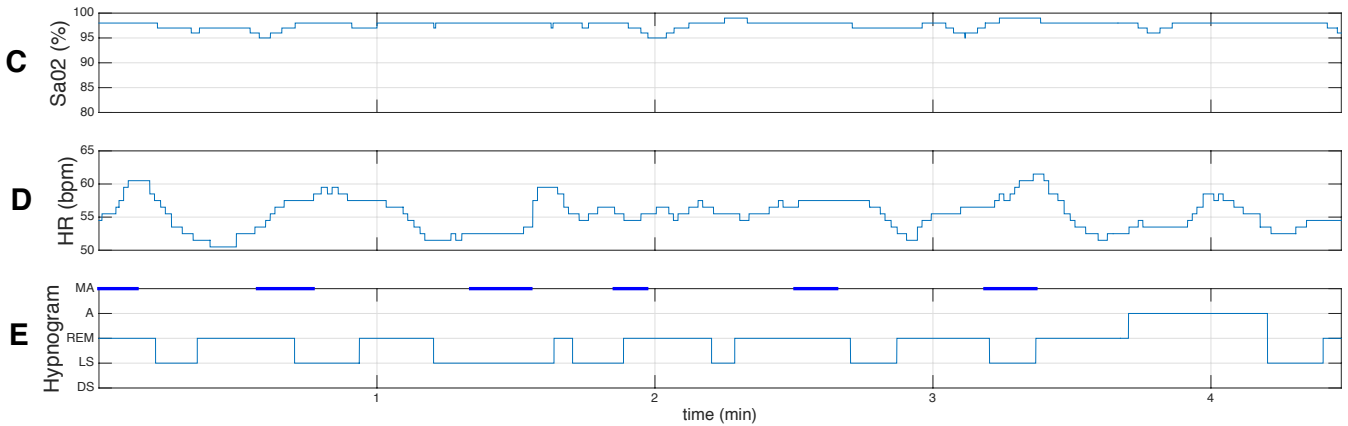

Figure 7: Cardiorespiratory response to kinesthetic stimulation of a non-responder patient. The whole segment was acquired during a stim-on phase. The following signals are displayed: A) nasal pressure (NP), B) signal driving the kinesthetic stimulator (Stim), C) oxygen saturation $\left.\left(\mathrm{SaO}_{2}\right), \mathrm{D}\right)$ instantaneous heart rate $(\mathrm{HR})$, as detected from the ECG,

E) Hypnogram obtained from Core-lab annotations ( $\mathrm{MA}=$ micro-arousal, $\mathrm{A}=\mathrm{Awake}$, $\mathrm{REM}=$ Rapid eye movement, $\mathrm{LS}=$ light sleep, $\mathrm{DS}=$ deep sleep). In this example the effect of the therapy is not clear. Although the respiratory events are of limited duration, most events are stimulated with the maximum of 3 stimulation bursts and normal respiration is not recovered just after the stimulation. 


\section{Discussion}

The PASITHEA v1 system worked satisfactorily during this first clinical evaluation. Some technical difficulties were observed during the titration phase, including Holter communication loss and the delivery of inappropriate stimulation bursts. These problems were corrected before including the 34 patients of the second study phase. During the second study phase, the only technical difficulties were related to a loss of continuity in the cable driving the kinesthetic actuator and the physical uncoupling of the actuator from the patients' skin.

Concerning respiratory event detection, the initial method presented by our team in [11], was improved and evaluated retrospectively. It should be noticed that the proposed method has been developed for a real-time control application, in order to activate the kinesthetic therapy. This real-time constraint means that the final detection has to be performed during the event (before the event ends and normal breathing resumes), which is not the case of most respiratory event detectors of the literature. Indeed, Berry et al. reported an overall sensitivity of $58 \%$ and specificity of $98 \%$ for a detector incorporated into their PAP system [14]. Contrary to our detector, they do wait for the end of an event before counting it as an event (post-event detection), which presumably accounts for their better specificity. Using oxygen desaturation and plethysmography as input signals, the detector proposed by Amir et al. showed a global sensitivity of $81 \%$ and a PPV of $81.4 \%$ [15]. Yet again, their detector counted events once terminated, since oxygen desaturation can occur between 5 and 60 seconds after the end of a hypopnea/apnea. Under the real-time constraints imposed by the PASITHEA project, the proposed, improved real-time respiratory event detector provides a satisfactory performance in terms of sensibility, specificity and, in particular, detection delay.

This paper also shows original results concerning triggered kinesthetic stimulation for the treatment of SAS. This first evaluation gave us valuable information on the feasibility of this therapy. Qualitative analysis of the effect of the therapy on responder patients showed how the acute cardiorespiratory consequences of respiratory events observed during the stim-off period (prolonged apnea, hypoxia events associated with tachy- and bradycardia), which may be deleterious to the patient in the long term, may be stopped or significantly attenuated during the stim-on phase (see for example Fig. 5). However, not all patients responded correctly to the therapy. We hypothesize 
that most of the non-responses observed on partially-responder patients may be avoided by i) an earlier detection of respiratory episodes (such as that obtained from the improved detector), leading to an earlier therapy; ii) appropriate adaptation and personalization of the stimulation parameters and iii) an improvement and dynamic estimation of the mechanical coupling between the actuator and the skin of the patient. Indeed, the control algorithm used in this first study was rather simple (on-off control) and the stimulation parameters (amplitude, burst duration, silent interval, maximum number of bursts) were empirically fixed for all patients using the analysis of the first 12 patients of the clinical study. The objective of the second phase of the PASITHEA project is to develop and evaluate such an advanced auto-adaptive and personalized therapy delivery. The information acquired on this first study and our recent works on algorithm-switching and model-based control design are particularly valuable for these developments [16][17]. Finally, like in any other therapy, it is expected that some patients will not respond to the proposed stimulation approach. Further studies are directed to identify the patients profile allowing for the optimization of patient selection.

\section{Conclusions and future work}

This paper presented an original ambulatory system for real-time detection and triggered therapy delivery, directed to patients suffering from SAS. A first evaluation of this system on 46 severe obstructive SAS patients has been performed, by assessing the ability of the system to correctly detect respiratory events in real-time. This study also provided preliminary results on the effect of the triggered kinesthetic stimulation therapy on these events.

Real-time respiratory event detection is clearly a challenge and the characteristic trade-off between high sensitivity and high PPV is accentuated by the real-time constraints. For the sake of the PASITHEA project, the aim is to detect as many events as possible, and as early as possible, in order to treat them before they can fully develop and result in desaturation or micro-arousal. The improved detector presented in this paper was designed for that purpose. The retrospective evaluation presented in this paper shows that the improved detector provides a satisfactory performance, approaching the level of performance of some post-event detectors.

Regarding the effects of kinesthetic stimulation on respiratory events, preliminary results shown in this work are very encouraging. In order to better characterize these effects on responder patients, a quantitative analysis is 
currently ongoing, by statistically comparing the duration and frequency of respiratory events during stimulation or non-stimulation periods. The sleep structure will also be quantitatively analyzed. Concerning non-responder patients, two major improvements of the system are currently in development: i) the replacement of the "on-off" control method by a fully-adaptive stimulation approach, based on multi-variate proportional-integral-derivative (PID) controllers and ii) the integration of new sensors to estimate the level of mechanical coupling of the actuator. Also, a better characterization the patient population that may benefit from the proposed kinesthetic therapy is being performed (e.g. patients without an autonomic dysfunction).

\section{Acknowledgments}

This project was supported by the French Research National Agency (ANR-12-TECS-0010 project). The authors thank F. Gagnadoux and the team of the sleep laboratory of Angers CHU, Y. Dauvilliers and his team in Montpellier CHU, Nadège Limousin and her team in Tours CHU, and all patients who agreed to participate to the study.

\section{References}

[1] P. Jennum, R. L. Riha, Epidemiology of sleep apnoea/hypopnoea syndrome and sleep-disordered breathing, Eur Respir J 33 (4) (2009) 90714.

[2] P. E. Peppard, T. Young, J. H. Barnet, M. Palta, E. Hagen, K. M. Hla, Increased prevalence of sleep-disordered breathing in adults, American Journal of Epidemiology 177 (9) (2013) 1006-1014, times Cited: 2404 241.

[3] T. D. Bradley, J. S. Floras, Obstructive sleep apnoea and its cardiovascular consequences, Lancet 373 (9657) (2009) 82-93.

[4] K. Todd, W. F. McIntyre, A. Baranchuk, Obstructive sleep apnea and atrial fibrillation, Nat Sci Sleep 2 (2010) 39-45.

[5] G. D. Foster, K. E. Borradaile, M. H. Sanders, R. Millman, G. Zammit, A. B. Newman, T. A. Wadden, D. Kelley, R. R. Wing, F. X. Pi-Sunyer, D. Reboussin, S. T. Kuna, A randomized study on the effect of weight 
loss on obstructive sleep apnea among obese patients with type 2 diabetes: the sleep ahead study, Arch Intern Med 169 (17) (2009) 1619-26.

[6] D. J. Gottlieb, G. Yenokyan, A. B. Newman, G. T. O'Connor, N. M. Punjabi, S. F. Quan, S. Redline, H. E. Resnick, E. K. Tong, M. DienerWest, E. Shahar, Prospective study of obstructive sleep apnea and incident coronary heart disease and heart failure the sleep heart health study, Circulation 122 (4) (2010) 352-360.

[7] L. J. Epstein, D. Kristo, P. J. Strollo, N. Friedman, A. Malhotra, S. P. Patil, K. Ramar, R. Rogers, R. J. Schwab, E. M. Weaver, M. D. Weinstein, M. Amer Acad Sleep, Clinical guideline for the evaluation, management and long-term care of obstructive sleep apnea in adults, Journal of Clinical Sleep Medicine 5 (3) (2009) 263-276.

[8] J. M. Marin, S. J. Carrizo, E. Vicente, A. G. N. Agusti, Long-term cardiovascular outcomes in men with obstructive sleep apnoea-hypopnoea with or without treatment with continuous positive airway pressure: an observational study, The Lancet 365 (9464) 1046-1053.

[9] W. Galetke, L. Puzzo, C. Priegnitz, N. Anduleit, W. J. Randerath, Long-term therapy with continuous positive airway pressure in obstructive sleep apnea: adherence, side effects and predictors of withdrawal a 'real-life' study, Respiration 82 (2) (2011) 155-61.

[10] A. Hernández, J. Cruz, G. Garrault, Device for supervision and stimulation intended to fight sleep apnea, WO Patent App. PCT/EP2007/055,723 (Dec. 13 2007).

[11] D. Feuerstein, L. Graindorge, A. Amblard, A. Tatar, G. Guerrero, S. Christophle-Boulard, C. Loiodice, A. I. Hernández, J. Pépin, Realtime detection of sleep breathing disorders, in: Computing in Cardiology Conference (CinC), 2015, Vol. 42, pp. 317-320.

[12] A. Amblard, L. Graindorge, D. Feuerstein, A. Hernández, J. Pépin, Système de traitement d'un trouble respiratoire par stimulation kinesthśique, avec contrôle de stabilisation de la stimulation, FR Patent App. FR-1462041 (Dec. 8 2014).

[13] A. Amblard, L. Graindorge, D. Feuerstein, A. Hernández, J. Pépin, Systèmeme de traitement d'un trouble respiratoire par stimulation 
kinesthésiquesique, avec sélection des stratégies de stimulation, FR Patent App. FR-1462039 (Dec. 8 2014).

[14] R. M. Berry, C. Kushida, M. Kryger, H. Soto-Calderon, B. Staley, S. Kuna, Respiratory event detection by a positive airway pressure device, Sleep 35 (3) (2012) 361-367.

[15] O. Amir, D. Barak-Shinar, A. Henry, F. Smart, Photoplethysmography as a single source for analysis of sleep-disordered breathing in patients with severe cardiovascular disease, J. Sleep Res. 21 (2012) 94-100.

[16] L. Giorgis, P. Frogerais, A. Amblard, E. Donal, P. Mabo, L. Senhadji, A. I. Hernández, Optimal algorithm switching for the estimation of systole period from cardiac microacceleration signals (sonr), IEEE Trans Biomed Eng 59 (11) (2012) 3009-15.

[17] H. Ugalde, D. Ojeda, V. Le Rolle, D. Andreu, D. Guiraud, J.-L. Bonnet, C. Henry, N. Karam, A. Hagege, P. Mabo, A. I. Hernández, Model-based design and experimental validation of control modules for neuromodulation devices, IEEE Trans Biomed Eng (2016) in press. 